\title{
INEQUALITIES SIMILAR TO THE INTEGRAL ANALOGUE OF HILBERT'S INEQUALITY
}

\author{
B. G. PACHPATTE
}

\begin{abstract}
In the present paper we establish some new inequalities similar to the integral analogue of Hilbert's inequality by using a fairly elementary analysis.
\end{abstract}

\section{Introduction}

The integral analogue of the well known Hilbert's double series theorem can be stated as follows (see [3, p.226]).

Theorem $\mathbb{H}$. If $p>1, p^{\prime}=p /(p-1)$ and

$$
\int_{0}^{\infty} f^{p}(x) d x \leq F, \int_{0}^{\infty} g^{p^{\prime}}(y) d y \leq G
$$

then

$$
\int_{0}^{\infty} \int_{0}^{\infty} \frac{f(x) g(y)}{x+y} d x d y<\frac{\pi}{\sin (\pi / p)} F^{1 / p} G^{1 / p^{\prime}}
$$

unless $f=0$ or $g=0$.

Since the discovery of the most celebrated Hilbert's double series theorem and its integral analogue given in Theorem $\mathrm{H}$, a large number of papers dealing with different proofs, numberous variants, various generalizations and their applications have appeared in the literature, see $[1-7,12]$. For the earlier historical development of this kind of inequalities and many important applications in analysis, see [3, Chapter IX]. Recently, in [8-11] the present author has established some new inequalities similar to Hilbert's series inequality and its integral analogue which we believe will serve as a model for further investigations. The main purpose of this paper is to establish some new inequalities similar to the integral analogue of the Hilbert's inequality involving functions and their higher order derivatives. The analysis used in the proofs is elementary and our results provide new estimates on inequalities of this type.

Received August 20, 1998.

1991 Mathematics Subject Classification. 26D10, 26D15.

Key words and phrases. Hilbert's inequality, integral analogue, Hilbert's double series theorem, Schwarz inequality. 


\section{Statement of Results}

In what follows we denote by $R$ the set of real numbers. Let $I_{x}=[0, x), I_{y}=[0, y)$, $x>0, y>0, I=[0, \infty), R_{+}=(0, \infty)$ denotes the intervals in $R$.

Our main results are given in the following theroems.

Theorem 1. Let $n \geq 1$ be an integer. Let $u \in C^{n}\left(I_{x}, R\right), v \in C^{n}\left(I_{y}, R\right)$ and $u^{(i)}(0)=v^{(i)}(0)=0$ for $i=0,1,2, \ldots, n-1$. Then

$$
\begin{aligned}
& \int_{0}^{x} \int_{0}^{y} \frac{\left|u^{(k)}(s)\right|\left|v^{(k)}(t)\right|}{s^{2 n-2 k-1}+t^{2 n-2 k-1}} d s d t \\
\leq & M_{1}(n, k, x, y)\left(\int_{0}^{x}(x-s)\left|u^{(n)}(s)\right|^{2} d s\right)^{1 / 2}\left(\int_{0}^{y}(y-t)\left|v^{(n)}(t)\right|^{2} d t\right)^{1 / 2},
\end{aligned}
$$

where

$$
M_{1}(n, k, x, y)=\frac{1}{2} \frac{\sqrt{x y}}{[(n-k-1) !]^{2}(2 n-2 k-1)} .
$$

Theorem 2. Let $n \geq 2,0 \leq k \leq n-2$ be integers. Let $r \in C^{1}\left(I, R_{+}\right), u \in C^{n}\left(I_{x}, R\right)$, $v \in c^{n}\left(I_{y}, R\right)$ and $u^{(i-1)}(0)=v^{(i-1)}(0)=0, i=1,2, \ldots, n$. Then

$$
\begin{aligned}
& \int_{0}^{x} \int_{0}^{y} \frac{\left|u^{(k)}(s)\right|\left|v^{(k)}(t)\right|}{s^{2 n-2 k-3}+t^{2 n-2 k-3}} d s d t \\
\leq & M_{2}(n, k, x, y)\left(\int_{0}^{x}(x-s) \frac{s}{r^{2}(t)}\left(\int_{0}^{s}\left|\left(r(\sigma) u^{(n-1)}(\sigma)\right)^{\prime}\right|^{2} d \sigma\right) d s\right)^{1 / 2} \\
& \left.\times\left(\left.\int_{0}^{y}(y-t) \frac{t}{r^{2}(t)}\left(\int_{0}^{t} \mid r(\xi) v^{(n-1)}(\xi)\right)^{\prime}\right|^{2} d \xi\right) d t\right)^{1 / 2}
\end{aligned}
$$

where

$$
M_{2}(n, k, x, y)=\frac{1}{2} \frac{\sqrt{x y}}{[(n-k-2) !]^{2}(2 n-2 k-3)} .
$$

Theorem 3. Let $n \geq 1,0 \leq k \leq n-1$ be integers. Let $r \in C^{n}\left(I, R_{+}\right)$, $u \in C^{2 n}\left(I_{x}, R\right), v \in C^{2 n}\left(I_{y}, R\right)$ and $u^{(i-1)}(0)=v^{(i-1)}(0)=0,\left(r(0) u^{(n)}(0)\right)^{(i-1)}=$
$\left(r(0) v^{(n)}(0)\right)^{(i-1)}=0, i=1,2, \ldots, n$. Then

$$
\begin{aligned}
& \int_{0}^{x} \int_{0}^{y} \frac{\left|u^{(k)}(s)\right|\left|v^{(k)}(t)\right|}{s^{2 n-2 k-1}+t^{2 n-2 k-1}} d s d t \\
\leq & M_{3}(n, k, x, y)\left[\int_{0}^{x}(x-s) \frac{s^{2 n-1}}{r^{2}(s)}\left(\int_{0}^{s}\left|\left(r(\sigma) u^{(n)}(\sigma)\right)^{(n)}\right|^{2} d \sigma\right) d s\right]^{1 / 2} \\
& \times\left[\int_{0}^{y}(y-t) \frac{t^{2 n-1}}{r^{2}(t)}\left(\int_{0}^{t}\left|\left(r(\xi) v^{(n)}(\xi)\right)^{(n)}\right|^{2} d \xi\right) d t\right]^{1 / 2},
\end{aligned}
$$

where

$$
M_{3}(n, k, x, y)=\frac{1}{2} \frac{\sqrt{x y}}{[(n-1) !(n-k-1) !]^{2}(2 n-1)(2 n-2 k-1)} .
$$


Remark 1 . We note that in the special case when $k=0$, the inequalities established in Theorems 1-3 reduces respectively to the following new inequalities

$$
\begin{aligned}
\int_{0}^{x} \int_{0}^{y} \frac{|u(s)||v(t)|}{s^{2 n-1}+t^{2 n-1}} d s d t \leq & M_{1}(n, 0, x, y)\left(\int_{0}^{x}(x-s)\left|u^{(n)}(s)\right|^{2} d s\right)^{1 / 2} \\
& \times\left(\int_{0}^{y}(y-t)\left|v^{(n)}(t)\right|^{2} d t\right)^{1 / 2}
\end{aligned}
$$

We note that in a recent paper [9] the author has proved the special version of the inequality given in Theorem 1 when $k=0$ and $n=1$.

\section{Proof of Theorem 1}

From the hypotheses and Taylor expansion we have

$$
\begin{aligned}
& u^{(k)}(s)=\frac{1}{(n-k-1) !} \int_{0}^{s}(s-\tau)^{n-k-1} u^{(n)}(\tau) d \tau \\
& v^{(k)}(t)=(t)=\frac{1}{(n-k-1) !} \int_{0}^{t}(t-\sigma)^{n-k-1} v^{(n)}(\sigma) d \sigma
\end{aligned}
$$

for $s \in I_{x}, t \in I_{y}$. From (7) and using Schwarz inequality we have

$$
\begin{aligned}
\left|u^{(k)}(s)\right| & \leq \frac{1}{(n-k-1) !} \int_{0}^{s}(s-\tau)^{n-k-1}\left|u^{(n)}(\tau)\right| d \tau \\
& \leq \frac{1}{(n-k-1) !}\left(\int_{0}^{s}(s-\tau)^{2(n-k-1)} d \tau\right)^{1 / 2}\left(\int_{0}^{s}\left|u^{(n)}(\tau)\right|^{2} d \tau\right)^{1 / 2} \\
& =\frac{1}{(n-k-1) !} \frac{s^{(2 n-2 k-1) / 2}}{(2 n-2 k-1)^{1 / 2}}\left(\int_{0}^{s}\left|u^{(n)}(\tau)\right|^{2} d \tau\right)^{1 / 2},
\end{aligned}
$$


for $s \in I_{x}$. Similarly, from (8) and using Schwarz inequality we have

$$
\left|v^{(k)}(t)\right| \leq \frac{1}{(n-k-1) !} \frac{t^{(2 n-2 k-1) / 2}}{(2 n-2 k-1)^{1 / 2}}\left(\int_{0}^{t}\left|v^{(n)}(\sigma)\right|^{2} d \sigma\right)^{1 / 2},
$$
for $t \in I_{y}$. From (9), (10) and using the elementary inequality $c^{1 / 2} d^{1 / 2} \leq \frac{1}{2}(c+d)$ (for
$c, d$ nonegative reals) we have

$$
\begin{aligned}
\left|u^{(k)}(s)\right|\left|v^{(k)}(t)\right| \leq & \frac{1}{2} \frac{1}{[(n-k-1) !]^{2}(2 n-2 k-1)}\left[s^{2 n-2 k-1}+t^{2 n-2 k-1}\right] \\
& \times\left(\int_{0}^{s}\left|u^{(n)}(\tau)\right|^{2} d \tau\right)^{1 / 2}\left(\int_{0}^{t}\left|v^{(n)}(\sigma)\right|^{2}\right)^{1 / 2},
\end{aligned}
$$

for $s \in I_{x}, t \in I_{y}$. Rewriting (11) and then integrating over $t$ from 0 to $y$ first and then integrating the resulting inequality over $s$ from 0 and $x$ and using Schwarz inequality we
have

$$
\begin{aligned}
& \int_{0}^{x} \int_{0}^{y} \frac{\left|u^{(k)}(s)\right|\left|v^{(k)}(t)\right|}{s^{2 n-2 k-1}+t^{2 n-2 k-1}} d s d t \\
\leq & \frac{1}{2} \frac{1}{[(n-k-1) !]^{2}(2 n-2 k-1)}\left[\int_{0}^{x}\left(\int_{0}^{s}\left|u^{(n)}(\tau)\right|^{2} d \tau\right)^{1 / 2} d s\right] \\
& \times\left[\int_{0}^{y}\left(\int_{0}^{t}\left|v^{(n)}(\sigma)\right|^{2} d \sigma\right)^{1 / 2} d t\right] \\
\leq & \frac{1}{2} \frac{1}{[(n-k-1) !]^{2}(2 n-2 k-1)} \sqrt{x}\left[\int_{0}^{x}\left(\int_{0}^{s}\left|u^{(n)}(\tau)\right|^{2} d \tau\right) d s\right)^{1 / 2} \\
& \left.\times \sqrt{y}\left(\int_{0}^{y}\left(\int_{0}^{t}\left|v^{(n)}(\sigma)\right|^{2} d \sigma\right) d t\right]\right]^{1 / 2} \\
= & M_{1}(n, k, x, y)\left(\int_{0}^{x}(x-s)\left|u^{(n)}(s)\right|^{2} d s\right)^{1 / 2} \times\left(\int_{0}^{y}(y-t)\left|v^{(n)}(t)\right|^{2} d t\right)^{1 / 2} .
\end{aligned}
$$

This completes the proof.

\section{Proof of Theorem 2}

From the hypotheses and Taylor expanson we have

$$
\begin{aligned}
r(s) u^{(n-1)}(s) & =\int_{0}^{s}\left(r(\tau) u^{(n-1)}(\tau)\right)^{\prime} d \tau \\
u^{(k)}(s) & =\frac{1}{(n-k-2) !} \int_{0}^{s}(s-\tau)^{n-k-2} u^{(n-1)}(\tau) d \tau,
\end{aligned}
$$

for $s \in I_{x}$. From (12) and (13) we observe that

$$
u^{(k)}(s)=\frac{1}{(n-k)-2) !} \int_{0}^{s}(s-\tau)^{n-k-2} \frac{1}{r(\tau)}\left(\int_{0}^{\tau}\left(r(\sigma) u^{(n-1)}(\sigma)\right)^{\prime} d \sigma\right) d \tau
$$


for $s \in I_{x}$. Similarly, we have

$$
v^{(k)}(t)=\frac{1}{(n-k-2) !} \int_{0}^{t}(t-\eta)^{n-k-2} \frac{1}{r(\eta)}\left(\int_{0}^{\eta}\left(r(\xi) v^{(n-1)}(\xi)\right)^{\prime} d \xi\right) d \eta,
$$

for $t \in I_{y}$. From (14) and using Schwarz inequality we have

$$
\begin{aligned}
\left|u^{(k)}(s)\right| \leq & \frac{1}{(n-k-2) !} \int_{0}^{s}(s-\tau)^{n-k-2}\left(\frac{1}{r(\tau)} \int_{0}^{\tau}\left|\left(r(\sigma) u^{(n-1)}(\sigma)\right)^{\prime}\right| d \sigma\right) d \tau \\
\leq & \frac{1}{(n-k-2) !}\left(\int_{0}^{s}(s-\tau)^{2(n-k-2)} d \tau\right)^{1 / 2} \\
& \times\left[\int_{0}^{s}\left(\frac{1}{r(\tau)} \int_{0}^{\tau}\left|\left(r(\sigma) u^{(n-1)}(\sigma)\right)^{\prime}\right| d \sigma\right)^{2} d \tau\right]^{1 / 2} \\
= & \frac{1}{(n-k-2) !} \frac{s^{(2 n-2 k-3) / 2}}{(2 n-2 k-3)^{1 / 2}} \times\left[\int_{0}^{s} \frac{1}{r^{2}(\tau)}\left(\int_{0}^{\tau}\left|\left(r(\sigma) u^{(n-1)}(\sigma)\right)^{\prime}\right| d \sigma\right)^{2} d \tau\right]^{1 / 2} \\
\leq & \frac{1}{(n-k-2) !} \frac{s^{(2 n-2 k-3) / 2}}{(2 n-2 k-3)^{1 / 2}}\left[\int_{0}^{s} \frac{1}{r^{2}(\tau)} \tau\left(\int_{0}^{\tau}\left|\left(r(\sigma) u^{(n-1)}(\sigma)\right)^{\prime}\right|^{2} d \sigma\right) d \tau\right]^{1 / 2},
\end{aligned}
$$

for $s \in I_{x}$. Similarly, we have

$$
\begin{aligned}
\left|v^{(k)}(t)\right| \leq & \frac{1}{(n-k-2) !} \frac{t^{(2 n-2 k-3) / 2}}{(2 n-2 k-3)^{1 / 2}} \\
& \times\left[\int_{0}^{t} \frac{1}{r^{2}(\eta)} \eta\left(\int_{0}^{\eta}\left|\left(r(\xi) v^{(n-1)}(\xi)\right)^{\prime}\right|^{2} d \xi\right) d \eta\right]^{1 / 2},
\end{aligned}
$$
for $t \in I_{y}$. Form (16) and (17) and using the elementary inequality $c^{1 / 2} d^{1 / 2} \leq \frac{1}{2}(c+d)$
(for $c, d$ nonegative reals) we have

$$
\begin{aligned}
\left|u^{(k)}(s) \| v^{(k)}(t)\right| \leq & \frac{1}{2} \frac{\left[s^{2 n-2 k-3}+t^{2 n-2 k-3}\right]}{[(n-k-2) !]^{2}(2 n-2 k-3)} \\
& \times\left[\int_{0}^{s} \frac{\tau}{r^{2}(\tau)}\left(\int_{0}^{\tau}\left|\left(r(\sigma) u^{(n-1)}(\sigma)\right)^{\prime}\right|^{2} d \sigma\right) d \tau\right]^{1 / 2} \\
& \times\left[\int_{0}^{t} \frac{\eta}{r^{2}(\eta)}\left(\int_{0}^{\eta}\left|\left(r(\xi) v^{(n-1)}(\xi)\right)^{\prime}\right| d \xi\right) d \eta\right]^{1 / 2}
\end{aligned}
$$

for $s \in I_{x}, y \in I_{y}$. Rewriting (18) and then integrating over $t$ from 0 to $y$ first and then integrating the resulting inequality over $s$ from 0 to $x$ and using Schwarz inequality we
have

$$
\begin{aligned}
& \int_{0}^{x} \int_{0}^{y} \frac{\left|u^{(k)}(s)\right|\left|v^{(k)}(t)\right|}{s^{2 n-2 k-3}+t^{2 n-2 k-3}} d s d t \\
\leq & \frac{1}{2} \frac{1}{[(n-k-1) !]^{2}(2 n-2 k-3)} \times\left(\int_{0}^{x}\left[\int_{0}^{s} \frac{\tau}{r^{2}(\tau)}\left(\int_{0}^{\tau}\left|\left(r(\sigma) u^{(n-1)}(\sigma)\right)^{\prime}\right|^{2} d \sigma\right) d \tau\right]^{1 / 2} d s\right)
\end{aligned}
$$




$$
\begin{aligned}
& \times\left(\int_{0}^{y}\left[\int_{0}^{t} \frac{\eta}{r^{2}(\eta)}\left(\int_{0}^{\eta} \mid\left(r(\xi) v^{(n-1)}(\xi)\right)^{\prime} d \xi\right) d \eta\right]^{1 / 2} d t\right) \\
\leq & \frac{1}{2} \frac{1}{[(n-k-2) !]^{2}(2 n-2 k-3)} \\
& \left.\times \sqrt{x}\left[\int_{0}^{x}\left(\left.\int_{0}^{s} \frac{\tau}{r^{2}(\tau)}\left(\int_{0}^{\tau} \mid r(\sigma) u^{(n-1)}(\sigma)\right)^{\prime}\right|^{2} d \sigma\right) d \tau\right) d s\right]^{1 / 2} \\
& \times \sqrt{y}\left[\int_{0}^{y}\left(\int_{0}^{t} \frac{\eta}{r^{2}(\eta)}\left(\int_{0}^{\eta}\left|\left(r(\xi) v^{(n-1)}(\xi)\right)^{\prime}\right|^{2} d \xi\right) d \eta\right) d t\right]^{1 / 2} \\
= & M_{2}(n, k, x, y)\left[\int_{0}^{x}(x-s) \frac{s}{r^{2}(s)}\left(\int_{0}^{s}\left|\left(r(\sigma) u^{(n-1)}(\sigma)\right)^{\prime}\right|^{2} d \sigma\right) d s\right]^{1 / 2} \\
& \times\left[\int_{0}^{y}(y-t) \frac{t}{r^{2}(t)}\left(\int_{0}^{t}\left|\left(r(\xi) v^{(n-1)}(\xi)\right)^{\prime}\right|^{2} d \xi\right) d t\right]^{1 / 2} .
\end{aligned}
$$

The proof is complete.

\section{Proof of Theorem 3}

From the hypotheses and Taylor expanson we have

$$
\begin{aligned}
u^{(k)}(s) & =\frac{1}{(n-k-1) !} \int_{0}^{s}(s-\tau)^{n-k-1} u^{(n)}(\tau) d \tau, \\
r(s) u^{(n)}(s) & =\frac{1}{(n-1) !} \int_{0}^{s}(s-\tau)^{n-1}\left(r(\tau) u^{(n)}(\tau)\right)^{(n)} d \tau,
\end{aligned}
$$

for $s \in I_{x}$. From (19) and (20) we boserve that

$u^{(k)}(s)=\frac{1}{(n-1) !(n-k-1) !} \int_{0}^{s}(s-\tau)^{n-k-1}\left[\frac{1}{r(\tau)} \int_{0}^{\tau}(\tau-\sigma)^{n-1} \times\left(r(\sigma) u^{(n)}(\sigma)\right)^{(n)} d \sigma\right] d \tau$,

for $s \in I_{x}$. Similarly, we have

$$
v^{(k)}(t)=\frac{1}{(n-1) !(n-k-1) !} \int_{0}^{t}(t-\eta)^{n-k-1}\left[\frac{1}{r(\eta)} \int_{0}^{\eta}(\eta-\xi)^{n-1} \times\left(r(\xi) v^{(n)}(\xi)\right)^{(n)} d \xi\right] d \eta
$$

for $t \in I_{y}$. From (21) and using Schwarz inequality we have

$$
\begin{aligned}
\left|u^{(k)}(s)\right| \leq & \frac{1}{(n-1) !(n-k-1) !} \int_{0}^{s}(s-\tau)^{n-k-1}\left[\frac{1}{r(\tau)} \int_{0}^{\tau}(\tau-\sigma)^{n-1}\left|\left(r(\sigma) u^{(n)}(\sigma)\right)^{(n)}\right| d \sigma\right] d \tau \\
\leq & \frac{1}{(n-1) !(n-k-1) !}\left(\int_{0}^{s}(s-\tau)^{2(n-k-1)} d \tau\right)^{1 / 2} \\
& \times\left(\int_{0}^{s} \frac{1}{r^{2}(\tau)}\left[\int_{0}^{\tau}(\tau-\sigma)^{n-1}\left|\left(r(\sigma) u^{(n)}(\sigma)\right)^{(n)}\right| d \sigma\right]^{2} d \tau\right)^{1 / 2} \\
\leq & \frac{1}{(n-1) !(n-k-1) !} \frac{s^{(2 n-2 k-1) / 2}}{(2 n-2 k-1)^{1 / 2}}\left[\int_{0}^{s} \frac{1}{r^{2}(\tau)}\left(\int_{0}^{\tau}(\tau-\sigma)^{2(n-1)} d \sigma\right)\right.
\end{aligned}
$$




$$
\begin{aligned}
& \left.\times\left(\int_{0}^{\tau}\left|\left(r(\sigma) u^{(n)}(\sigma)\right)^{(n)}\right|^{2} d \sigma\right) d \tau\right]^{1 / 2} \\
= & \frac{1}{(n-1) !(n-k-1) !(2 n-1)^{1 / 2}} \frac{s^{(2 n-2 k-1) / 2}}{(2 n-2 k-1)^{1 / 2}} \\
& \times\left[\int_{0}^{s} \frac{\tau^{2 n-1}}{r^{2}(\tau)}\left(\int_{0}^{\tau}\left|\left(r(\sigma) u^{(n)}(\sigma)\right)^{(n)}\right|^{2} d \sigma\right) d \tau\right]^{1 / 2},
\end{aligned}
$$

for $s \in I_{x}$. Similarly, we have

$$
\begin{aligned}
\left|v^{(k)}(t)\right| \leq & \frac{1}{(n-1) !(n-k-1) !(2 n-1)^{1 / 2}} \frac{t^{(2 n-2 k-1) / 2}}{(2 n-2 !-1)^{1 / 2}} \\
& \times\left[\int_{0}^{t} \frac{\eta^{2 n-1}}{r^{2}(\eta)}\left(\int_{0}^{\eta}\left|\left(r(\xi) v^{(n)}(\xi)\right)^{(n)}\right|^{2} d \xi\right) d \eta\right]^{1 / 2}
\end{aligned}
$$

for $t \in I_{y}$.

The rest of the proof can be completed by following the same arguments as in the proof of Theorem 2 below the inequality (17) with suitable modifications. We leave the completion of the further proof to the reader.

Remark 2. We note that the bounds obtained on the right hand sides in (1), (3), (5) are different from the bound given in the integral analogue of the Hilbert's inequality given in Theorem $H$. Further, if we apply the elementary inequality $c^{1 / 2} d^{1 / 2} \leq \frac{1}{2}(c+d)$ (for $c, d$ nonnegative reals) on the right hand sides of $(1),(3),(5)$, then we get respectively the following new inequalities

$$
\begin{aligned}
& \int_{0}^{x} \int_{0}^{y} \frac{\left|u^{(k)}(s)\right|\left|v^{(k)}(t)\right|}{s^{2 n-2 k-1}+t^{2 n-2 k-1}} d s d t \\
\leq & \frac{1}{2} M_{1}(n, k, x, y)\left[\int_{0}^{x}(x-s)\left|u^{(n)}(s)\right|^{2} d s+\int_{0}^{y}(y-t)\left|v^{(n)}(t)\right|^{2} d t\right], \\
& \int_{0}^{x} \int_{0}^{y} \frac{\left|u^{(k)}(s)\right|\left|v^{(k)}(t)\right|}{s^{2 n-2 k-3}+t^{2 n-2 k-3}} d s d t \\
\leq & \frac{1}{2} M_{2}(n, k, x, y)\left[\int_{0}^{x}(x-s) \frac{s}{r^{2}(s)}\left(\int_{0}^{s}\left|\left(r(\sigma) u^{(n-1)}(\sigma)\right)^{\prime}\right|^{2} d \sigma\right) d s\right. \\
& \left.+\int_{0}^{y}(y-t) \frac{t}{r^{2}(t)}\left(\int_{0}^{t}\left|\left(r(\xi) v^{n-1)}(\xi)\right)^{\prime}\right|^{2} d \xi\right) d t\right], \\
& \int_{0}^{x} \int_{0}^{y} \frac{\left|u^{(k)}(s)\right|\left|v^{(k)}(t)\right|}{s^{2 n-2 k-1}+t^{2 n-2 k-1} d s d t} \\
\leq & \frac{1}{2} M_{3}(n, k, x, y)\left[\int_{0}^{x}(x-s) \frac{s^{2 n-1}}{r^{2}(s)}\left(\int_{0}^{s}\left|\left(r(\sigma) u^{(n)}(\sigma)\right)^{(n)}\right|^{2} d \sigma\right) d s\right. \\
& \left.+\int_{0}^{y}(y-t) \frac{t^{2 n-1}}{r^{2}(t)}\left(\int_{0}^{t}\left|\left(r(\xi) \mid v^{(n)}(\xi)\right)^{(n)}\right|^{2} d \xi\right) d t\right] .
\end{aligned}
$$

For various other inequalities different from those of given here, see [8-11]. 


\section{B. G. PACHPATTE}

\section{References}

[1] Yang Bicheng, On Hilbert's integral inequality, J. Math. Anal. Appl. 220(1998), 778-785. [2] Y. C. Chow, On inequalities of Hilbert and Widder, J. London Math. Soc. 14(1939),
151-154.

[3] G. H. Hardy, J. E. Littlewood and G. Polya, Inequalities, Cambridge University Press, 1934.

[4] V. Levin, On the two-parameter extension and analogue of Hilbert's inequality, J. London
Math. Soc. 11 (1936), 119-124.

[5] G. Mingze, An improvement of hardy-Riesz's extension of the Hilbert inequality, J. Math. Res. Exposition 14(1994), 255-259.

[6] G. Mingze, On Hilbert's inequality and its applications, J. Math. Anal. Appl. 212(1997),

[7] D. S. Mitrinović and J. E. Pečarić, On inequalities of Hilbert and Widder, Proc. Edinburgh Math. Soc. 34(1991), 411-414.

[8] B. G. Pachpatte, On some new inequalities similar to Hilbert's inequality, J. Math. Anal. Appl. 224(1998), to appear.

[9] B. G. Pachpatte, A note on an inequality of Hilbert type, Demonstratio Math. 32(1999), to
appear.

[10] B. G. Pachpatte, A note on Hilbert type inequality, Tamkang J. Math. 29(1998), to appear. [11] B. G. Pachpatte, On an inequality similar to Hilbert's inequality, Bull. Inst. Polyt. Iasi
Math., to appear.

[12] D. V. Widder, An inequality related to one of Hilbert's, J. London Math. Soc. 4(1929),
194-198.

Department of Mathematics, Marathwada University, Aurangabad 431 004, (Maharashtra) In-
dia. 